\title{
Efficient VLSI Architecture for ECG Data Compression
}

\author{
Ruqaiya Khanam \\ Department of Electronics \& Communication \\ Engineering \\ Jamaia Millia Islamia University \\ Delhi,INDIA
}

\begin{abstract}
This paper presents an efficient ECG signals compression techniques using a 2D DWT coefficient thresholding and its design implementation of an efficient JPEG2000 encoder that employs the Distributed Arithmetic (DA) technique for the complex computation of Discrete Wavelet Transform (DWT).2D approaches exploit the fact that redundancy of ECG signal occurs between adjacent beats and adjacent samples. Cut and aligned error signal to form 2D matrix, then 2D matrix is transformed. Ultimately wavelet coefficients are segmented into groups and thresholded. DA-DWT is used for reducing the complex computations, which can increase the speed and throughput as well. Architecture is based on the principles of pipelining and parallelism to obtain the optimal speed and throughput. Architecture is simple, modular and cascadable for computing a DA-DWT. This technique is faster when ROM table in on chip memory and memory size is reduced by splitting ROM table. The description and functionalities of the design is modeled by Verilog HDL. The simulation and synthesis methodology are used to target it on Virtex-II Pro FPGA (xc4v1x25-12sf363) that consumes 6\% resources of FPGA and shows the clock frequency $310.207 \mathrm{MHz}$ by DADWT using Sym20. Experiments on selected records from MIT-BIH arrhythmia database revealed that the proposed design is significantly more efficient.
\end{abstract}

\section{Keywords}

Electrocardiogram, Discrete Wavelet Transform, Distributed Arithmetic

\section{INTRODUCTION}

The extensive use of electrocardiogram (ECG) produces large amount of data. Since the need for ECG signal compression is two reasons: effective storage and effective real time transmission. The efficient compression techniques are important to reduce transmission time or required storage capacity of large data records. Several ECG compression methods have been developed during last three decades [1].

(1) Direct Methods-e.g.-AZTEC [2], DPCM, TP, CORTES, Scan-along polygonal approximation (SAPA) [3], FAN [4].

(2)Transform Methods- e.g.- Fourier, Walsh, KLT, Discrete Cosine Transform (DCT) [5], and Wavelet Transform (WT) [6][7][8].

\author{
Syed Naseem Ahmad \\ Department of Electronics \& Communication \\ Engineering \\ Jamaia Millia Islamia University \\ Delhi,INDIA
}

(3) Parameter Extraction Methods- e.g.- Vector Quantization (VQ) [9], Neural Network (NN),Prediction .

Our approach belongs to the second category. Usually, transform techniques involve expanding a signal into a and weighted sum of basis functions. The coefficients of this sum are properly encoded and stored or transmitted instead of the original data. Transform techniques include several waveletbased compression methods. Due Time-frequency localization properties of wavelets are better hence suitable for data compression applications.

Most of the methods process the heart beat data in 1-D form although some of these methods utilize redundancies between adjacent beats. Since the ECG signals has both intra-beat (sample to sample) and inter-beat (beat to beat) correlations, many 2-D compression approaches have been proposed for better compression performances. For example, Lee[5] used "cut and align beats approach and 2D discrete cosine transform" to obtain better compression results in regular ECG.Wei [11] used truncated singular value decomposition algorithm to compress 2D ECG arrays. Recently, wavelets are widely used for both $1 \mathrm{D}$ and 2D compression [6][7][8]. Due to better performance of data compression, choose JPEG2000 [12] as a codec.DWT has the high compression ratio and is free from blocking artifacts at low bit rates [13].

In this paper, the following paper sections: section II describes the 1-D to 2-D process. Section III presents the proposed DA-DWT based architecture for JPEG2000 encoder. In section IV, the performance analysis and implementation results are given. Finally, the conclusion is presented in section $\mathrm{V}$.

\section{2-D ECG PROCESS}

2-D compression methods comprises the following steps:

1) QRS Detection

2) Preprocessing (cut and align beats, period normalization, amplitude normalization, mean removal)

3) Transformation

4) Coefficient encoding

\subsection{QRS detection and alignment}

ECG signal is comprised from three main components, namely, the QRS complexes, P-wave and T-wave. As the QRS complexes have a time-varying morphology, strong 
signal component in an ECG signal is not necessary at all time. Hence the QRS complex characteristic same to that of the $\mathrm{P}$-waves or $\mathrm{T}$-waves as well as spikes from high frequency pacemakers can compromise the detection of the QRS complex.QRS detector must be robust for different noises and also able to detect QRS complexes in spite of the morphology of the ECG signal is varying with time.The detection algorithm is a MATLAB based detector[19]. Primarily the peaks of QRS waves should be selected to identify each heartbeat. After identify each QRS peak of heartbeat segments is identified, the original signal 1-D ECG signals are cut at every $130^{\text {th }}$ sample before each QRS peak (a $130^{\text {th }}$ sample shift from the QRS peak, a smooth region estimated from human physiology)[14].

\subsection{Period of heartbeats restriction}

The periods of heartbeats are not the same. Therefore the length of the heartbeats segments can be varied. If the ECG is found abnormal or the QRS peak detection misses or some QRS peaks identified heartbeat is very long then the size of 2-D array increases, this is the more dangerous situation. To achieve maximum data compression used an efficient compression scheme needs to utilize both dependencies. The length of detected heartbeats should be restricted to form of a 2-D ECG representation at moderate size. After the detection of QRS complex, each period is stored in 2D matrix. Here the intrabeat dependencies are in horizontal direction of the matrix and interbeat dependencies are in vertical direction. Fig. 1 shows the generated image .Due to the variation in length, the generated $2 \mathrm{D}$ ECG matrix will be using different number of data points in every row. Researchers have tried to overcome this problem. The compression system using JPEG2000 normalized each ECG period for equal length[16].

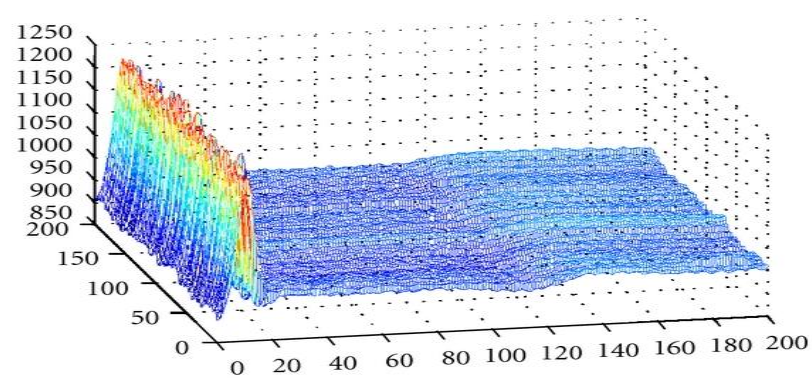

Fig.1 ECG 2D Matrix

\section{DISTRIBUTED ARITHMETIC- DISCRETEWAVELET TRANSFORM}

One major advantage opened by wavelets is the ability to perform local analysis, to analyze a localized area of a larger signal. Discrete Wavelet Transform allows the change of a signal from a time domain to a frequency -time domain through a decomposition and reconstruction process, the last one to recover the original signal[15].In the decomposition, the wavelet coefficients of any stage can be calculated from DWT of the previous stage using the following iterative equations:

$$
\begin{aligned}
& w_{L}(n, j)=\sum_{m} W_{L}(m, j-1) h_{0}(m-2 n) \\
& w_{H}(n, j)=\sum_{m} W_{L}(m, j-1) h_{1}(m-2 n)
\end{aligned}
$$

Where $W_{L}(n, j)$ is the $n$th scaling coefficient at the jth stage, $\mathrm{W}_{\mathrm{H}}(\mathrm{n}, \mathrm{j})$ is the nth wavelet coefficient at the jth stage, and $\mathrm{h}_{0}$ (n) and $h_{1}(n)$ are the dilation coefficients corresponding to the scaling and wavelet functions, and $\mathrm{m}$ is the summation running index of the analysis filter's coefficients, respectively.

In order to reconstruct the original data, the DWT coefficients are upsampled and passed through another set of low pass and high pass filters, which is expressed as follows:

$$
W_{L}(n, j)=\sum_{k} W(k, j+1) g_{0}(n-2 k)+\sum_{I} W_{H}(I, j+1) g_{1}(n-2 I)
$$

Where $g_{0}(n)$ and $g_{1}(n)$ are respectively the low-pass and high-pass synthesis filters corresponding to the mother wavelet, and 1 is summation running index of the analysis filter's coefficients. It is observed from equation(3) that the $j^{\text {th }}$ level coefficients can be obtained from the $(j+1)^{\text {th }}$ level coefficients.

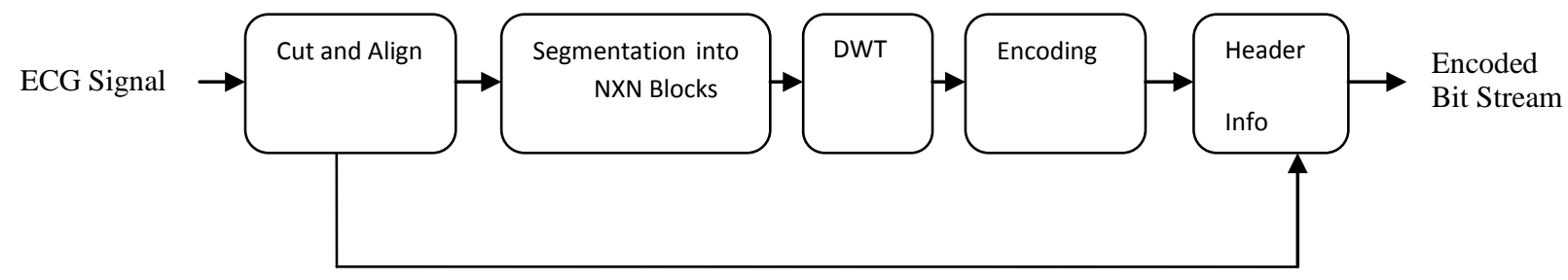

Fig.2 Block diagram of a WT-based ECG compression method 


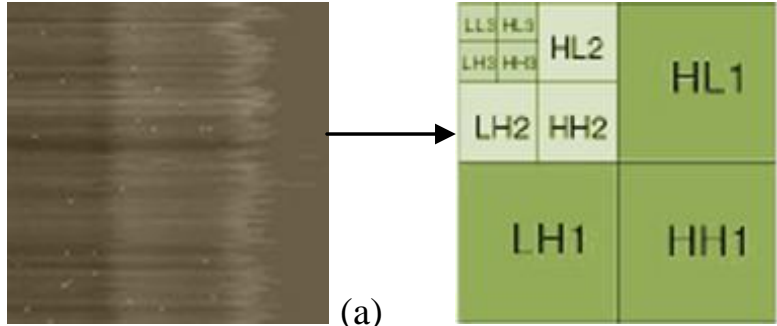

Original Image
Wavelet Transform Image

\section{$10101110 \ldots$.}

(b)

Bit Stream

Fig.3 Data representations after image reception, Wavelet transformation and encoding

The multimedia signals are stored or transmitted in compressed form to save time and resource. Efficient coding standards like MP3, JPEG2000 and MPEG4 are developed for multimedia signal.

The architecture has implemented DA-LUT using adders and multiplexers. Using this architecture the hardware and time complexity can be optimized.

To derive Distributed Architecture for DWT, consider the following sum of products:

$$
Y=\sum_{k=1}^{L} A_{k} X_{k}
$$

Where $A_{k}$ is the fixed coefficient of the filter bank and $X_{k}$ is the input samples. The decomposed equation of (4) in form of DA can be written as equation 2:

$$
\begin{gathered}
Y=\left[A_{1} A_{2} \ldots \ldots A_{L}\right]\left[\begin{array}{c}
X_{1} \\
X_{2} \\
\cdot \\
\cdots \\
\dot{X_{L}}
\end{array}\right] \\
=\left[2^{0} 2^{1} \ldots\left(-2^{N-1}\right)\right] A\left[\begin{array}{c}
X_{1} \\
X_{2} \\
\cdot \\
\cdot \\
\dot{X_{L}}
\end{array}\right]
\end{gathered}
$$

Where:

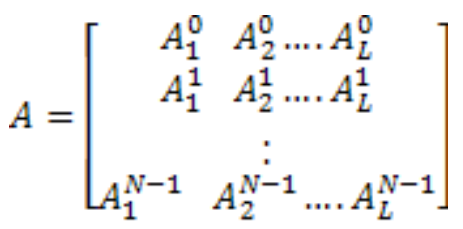

From equation (5), $\mathrm{A}$ is the distributed arithmetic matrix of fixed coefficients $A_{k}^{i}$, where $\mathrm{k}=1.2, \ldots, \mathrm{L} ; \mathrm{i}=1,2, \ldots, \mathrm{N}-1$, with $A_{k}^{N-1}$ is the MSB and $A_{k}^{0}$ is the LSB. In DA-DWT, the bits of the coefficients are distributed unlike conventional DA, where the bits of the input data words are distributed.
Distributed architecture matrix contains only 0 and 1 , which means the computation of $\mathrm{Y}$ can be obtained just by shifting and adding of the input vectors. The architecture of DA-DWT can save hardware to implement the computations.
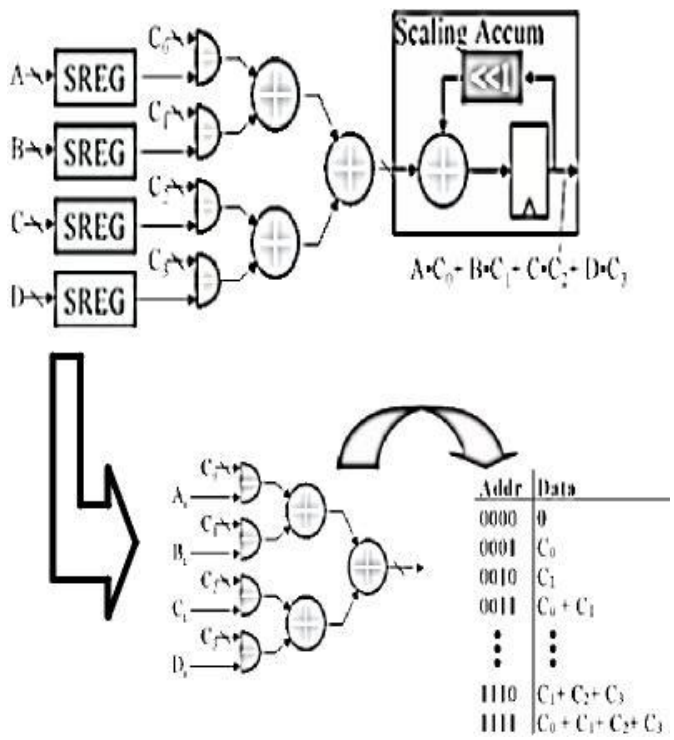

Fig.4 Bit level arrangement for DA MAC

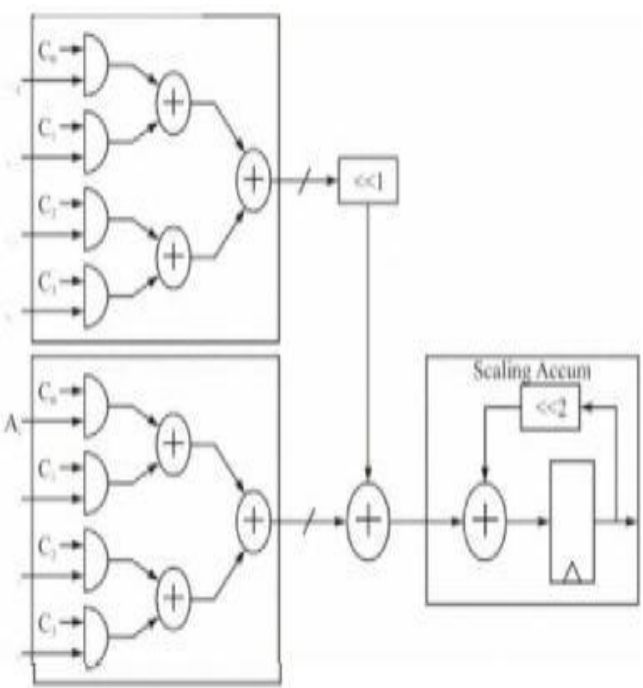

Fig. 5 Parallel implementation of DA technique 


\section{DA-DWT ARCHITECTURE IMPLEMENTED ON FPGA}

\section{Algorithm}

\section{BEGIN}

Step 1: Loading of original signal The signal which is taken from MIT-BIH arrhythmia data base

Step 2: Detect QRS complex in every heart beat

Step 3: Cut and align the signal beat by beat prior to 2-D transformation

Step 4: Segmentation into NXN blocks (to construct 2-D array from 1-D array)

Step 5: Apply DWT to get the transformed information using by DA-DWT architecture

Step 6: Find the transformed coefficients

Step 7: Apply 2-D transform coding to achieve the essential compression.

Step 8: Displaying the result

END

\section{Algorithm 2}

Step 1.The samples of input ECG signal are divided into even and odd in the first stage.

Step 2.Using shift register, upper four shift register store MSB bits and lower four shift register stores the LSB bits.
Step 3.Clock cycles are required to load the shift register contents. After clock cycle, the control logic configures the shift register as serial in parallel out, forming the address for the LUT.

Step 4.Partial products stored in the LUT are read simultaneously front all the four LUTs.

Step 5.Accumulated with previous values available across the shift register in the output stage.

Step 6. The output stage consisting of adders, accumulators and shift registers are used to accumulate the LUT contents and compute the DWT output.

Distributed Arithmetic replaces multiplications by ROM lookup table so that DWT filter can achieve high computing speed with small area by $80 \%$ in hardware design.

\section{SIMULATION AND SYNTHESIS METHODOLOGY}

ECG data compression proposed here is tested for record 117 from MIT-BIH arrhythmia database using by MATLAB

DA-DWT was described in Verilog HDL. The architecture was divided into blocks level and blocks are divided into sub blocks. At sub block level, behavioral description was made and at block level, structural description connecting the sub blocks was constructed and validated using the Xilinx software.
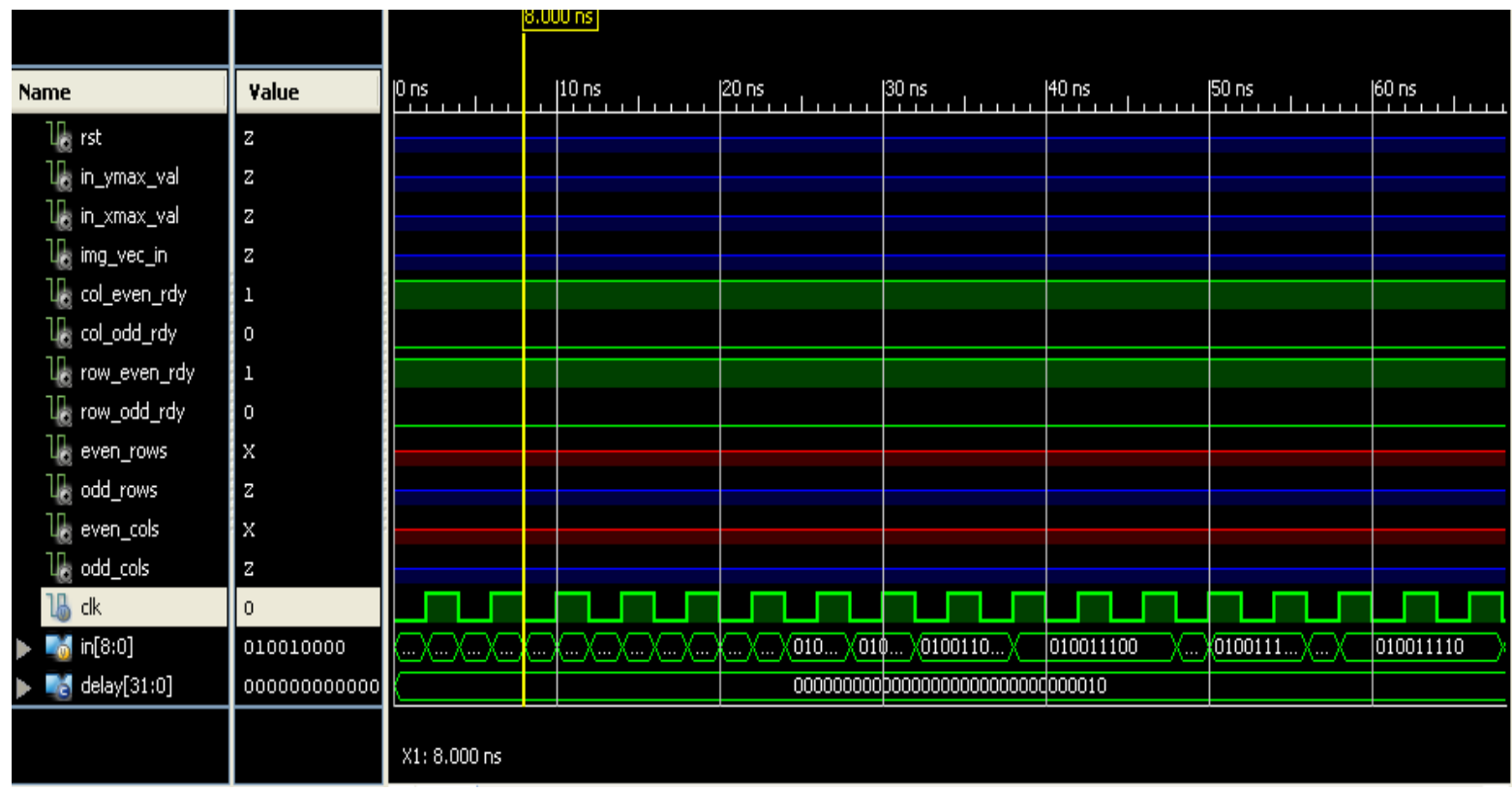

Fig. 6 Snapshot of the simulation of DWT

Fig. 6 shows the simulation result of DA-DWT and Fig.7 is also shows the synthesis result of this architecture. 


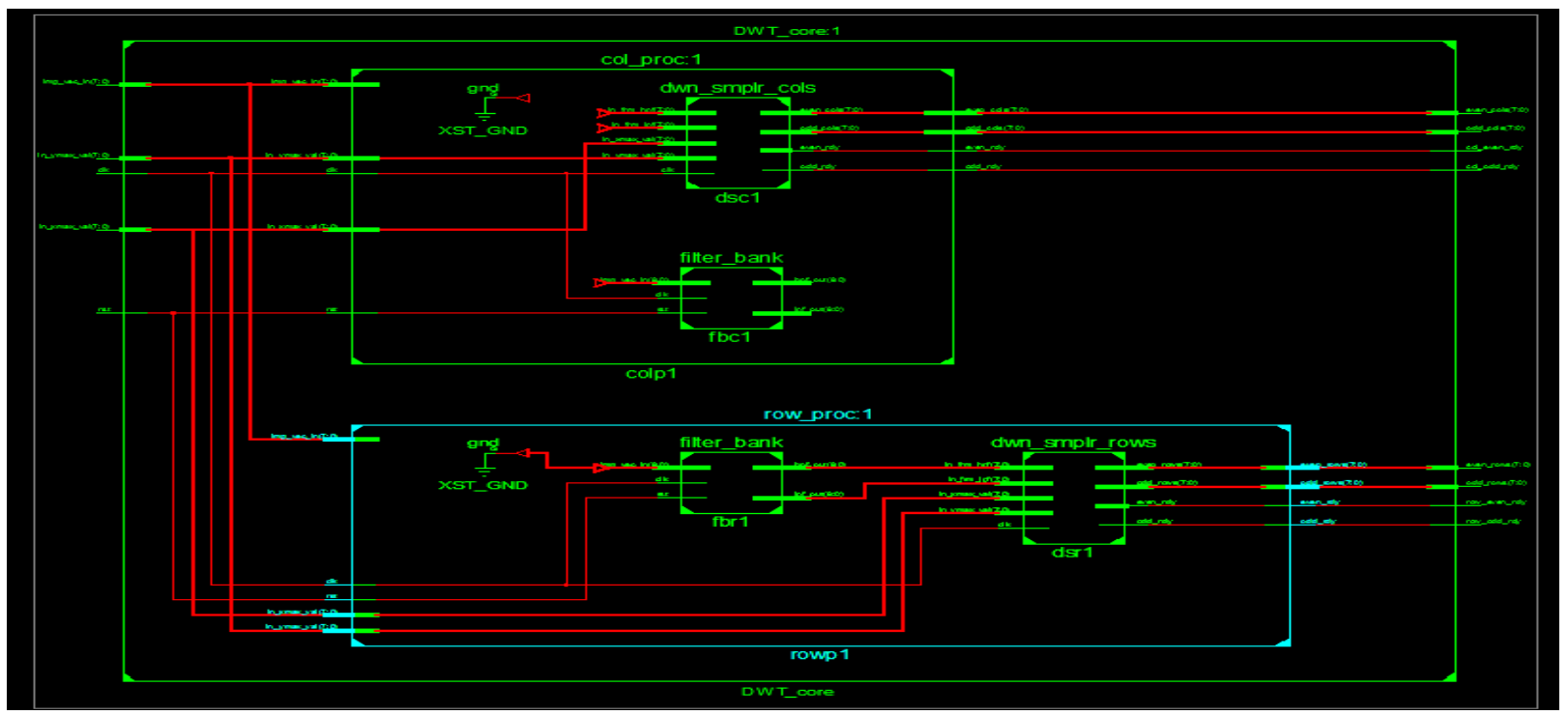

Fig. 7 Snapshot of RTL view of DWT

\section{EXPERIMENTAL RESULT}

Table 1 Synthesis Results of DA-DWT

\begin{tabular}{|c|c|c|}
\hline S.No & Devices & No. of Devices \\
\hline 1 & ROMs(2048x16-bit ROM) & 4 \\
\hline 2 & $\begin{array}{l}\text { Adder/Subtractors } \quad(9 \quad \text { bit } \\
\text { Adder })\end{array}$ & 26 \\
\hline 3 & $\begin{array}{l}\text { Counters (3-bit and 9-bit up } \\
\text { counter) }\end{array}$ & 2,2 \\
\hline 4 & $\begin{array}{l}\text { Registers (1-bit reg.,16-bit,8- } \\
\text { bit,9-bit regs.) }\end{array}$ & $32,28,4,2(66)$ \\
\hline 5 & Comparators & 10 \\
\hline 6 & Multiplexers & 4 \\
\hline 7 & Tristates & 4 \\
\hline
\end{tabular}

Table 2 Performance Comparison

\begin{tabular}{|c|c|c|}
\hline Scheme & Level-1 & Efficiency \\
\hline $\begin{array}{c}\text { Filter based } \\
\text { implementation }\end{array}$ & $16 \mathrm{x}$ Multiplier & $\mathrm{N}^{2}$ \\
\hline $\begin{array}{c}\text { Lifting Based } \\
\text { implementation }\end{array}$ & $6 \mathrm{x}$ Multiplier & $\mathrm{N}^{2}$ \\
\hline $\begin{array}{c}\text { Proposed } \\
\text { architecture }\end{array}$ & $26 \mathrm{x}$ Adders & $\mathrm{N}^{2} / 2$ \\
\hline
\end{tabular}

Above table 1 shows the performance of various architectures. The proposed architecture was obtained throughput by factor 2 than previous counterparts. The efficiency cycles were required to compute DWT of $\mathrm{NxN}$ image at same clock frequency.

\section{CONCLUSION}

In this paper, design gives faster results using highly parallel Virtex field programmable gate array devices (FPGA).The proposed architecture has been correctly verified by the Verilog Hardware Descriptive Language (Verilog HDL). DADWT is better area optimization and increase the throughput. In this architecture achieve the clock frequency upto 310.207 MHZ and area utilization are reduced by $7 \%$ lesser then the normal approach. Simulation and synthesis results are obtained in figure 3 and figure 4 respectively.

\section{REFERENCES}

[1] Jalaleddine S.M.S, Hutchen C.G, Strattan R.D. and Coberly W.A ,April 1990, ECG data Compression Techniques- A unified approach, IEEE Trans. On biomed.Eng., 37(4):329-343.

[2] Cox J.R., Noile F.M., Fozzard H.A., and Olover G.C, 1968, AZTEC : preprocessing program for real time ECG rhythm analysis, IEEE Trans. Biomed.Eng., vol. 15 , pp. 28-129.

[3] Ishijima M., Shin,S.B. Hostetter G.S., and Sklansky J., 1983, Scan-along polygonal approximation for data compression of electrocardiograms, IEEE Trans.Biomed. Eng., vol. 30, 11, pp. 723-729.

[4] Barr R.C., 1988 ,Adaptive sampling of cardiac waveforms, J. Electrocard, 21,pp. 57-60. 
[5] Lee H. and Buckley K.M., 1999 ,ECG data compression using cut and align beats approach and 2-D Transforms, IEEE Trans. Biomed. Eng., vol. 46, pp.556-565.

[6] Rajoub B.A., 2002,An efficient coding algorithm for the compression of ECG signal $s$ using the wavelet transform, IEEE Trans. Biomed. Eng.,vol. 49, pp. 355362.

[7] Hilton M.L, 1997, Wavelet and Wavelet packet compression of electrocardiograms, IEEE Trans Biomed. Eng., vol. 44, pp. 394-402.

[8] Moghaddam A.R.A and Nayebi K, 2001,A two dimensional wavelet packet approach for ECG compression, Signal processing and its applications, the 6th International, Symposium, pp.226-229.

[9] Wang B. and Yuan G, 1997,Compression of ECG data by vector quantization, IEEE Trans. Biomed. Eng., vol. 40,pp. 23-26.

[10] Nave G. and Cohen A, 1993,ECG compression using long-term prediction, IEEE Trans. Biomed. Eng., vol.40, pp. 877-885.

[11] Wei J.J, Chang C.J, Chou N.K, and Jan G.J, 2001ECG data compression using truncated singular value decomposition, IEEE Trans. Biomed. Eng., vol. 5, pp. 290-299.

[12] Morteza M.G, Taheri A, and Pooyan M, 2005,Efficient method for ECG compression using two dimensional Multiwavelet transform, An International journal of information technology, vol.2,4.

[13] Mohammadpour T.I and Mollaei M.R.K, 2009,ECG compression with thresholding of 2D wavelet transform oefficients and run length coding, European journal of scientific research, vol.27,no.2,pp.248-257.
[14] Tai S.C, Sun C.C and Yan W.C, 2005, A 2-D ECG compression method based on wavelet transform and modified SPIHT,IEEE Transactions on Biomedical Engineering, vol.52, no. 6,pp.999-1008.

[15] Sezgin M and Sankur B, 2004, Survey over image thresholding techniques and quantitative performance evaluation, Journal of Electronic Imaging, vol.13,no.1,pp.146-168.

[16] Bilgin A,Marcellin M.W and Altbach M.I,2003,Compression of electrocardiogram signals using JPEG2000, IEEE Transaction on consumer electronics, vol.49,no.4,pp.833-840.

[17] Zhang Qihui, Meng Nan, 2nd-5th Aug 2009 ,A low area pipelined 2D DCT architecture for JPEG encoder, MWSCAS'09,52nd IEEE international Midwest Symposium on Circuit and Systems, pp.747-750 Nagabushanam M., Raj P.C.P, Ramachandran,S.10-12 Feb.2011,Design and FPGA implementation of modified Distributed Arithmetic based DWT-IDWT processor for image compression,International conference on communications and signal processing (ICCSP), pp.1-4.

[18] Vander J, Sherman J.H , Luciano D.S, 1994,Human Physiology, McGraw-Hill, 6th edition, chap 14,pp393472 .

[19] Ruqaiya Khanam, Syed Naseem Ahmad, ,23-24 June 2012 , Peak Detection of ECG Signal Using Wavelet Transform" ,International Conference on Emerging Trends in Engineering \& Management (ICETEM122012),pp.200-203.

[20] Mallat S, 1999, A Wavelet Tour of signal processing. Academia Press.

[21] MIT-BIH Arrhythmia Database, http://www.physionet.org/physiobank/database/mitdb/. 